\title{
EFEITO DO MOLIBDÊNIO APLICADO VIA FOLIAR E VIA SEMENTES SOBRE O POTENCIAL FISIOLÓGICO E PRODUTIVIDADE DE SEMENTES DE SOJA ${ }^{1}$
}

\author{
JEAN CARLO POSSENTI2; FRANCISCO AMARAL VILLELA ${ }^{3}$
}

\begin{abstract}
RESUMO - Objetivou-se avaliar a qualidade fisiológica e a produtividade de sementes de soja produzidas a partir de sementes enriquecidas com molibdênio, via foliar e via sementes, em dois anos consecutivos. No primeiro ano, sementes de três cultivares, CD-206, MSOY-7101 e BRS-

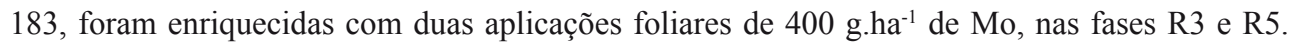
Avaliou-se a produtividade, peso de mil sementes e teor de molibdênio. A germinação e o vigor por envelhecimento acelerado foram avaliados aos zero e seis meses de armazenamento. No segundo ano, as cultivares CD-206 e BRS-183, produzidas a partir das sementes enriquecidas no ano anterior com Mo foram submetidas à aplicação, via semente, do elemento na dose de 20 g.ha- ${ }^{-1}$. Avaliou-se a produtividade, peso de mil sementes, teor de molibdênio, germinação e vigor por envelhecimento acelerado. A semente produzida mediante a aplicação de molibdênio em altas doses durante as fases de R3 e R5 apresenta altas concentrações do nutriente, porém, não transfere a mesma concentração do nutriente para a semente da próxima geração. Este enriquecimento e a aplicação via semente não afetam a qualidade fisiológica das sementes produzidas, tampouco a produtividade, nas condições em que o trabalho foi realizado.
\end{abstract}

Termos para indexação: Glycine max, micronutriente, rendimento, vigor.

\section{THE EFFECT OF MOLYBDENUM APPLIED BY FOLIAR SPRAYING AND SEED TREATMENT ON THE PHYSIOLOGICAL POTENTIAL AND PRODUCTIVITY OF SOYBEAN SEEDS}

\begin{abstract}
The objective of this study was to evaluate the physiological quality and productivity of soybean seeds produced from seeds enriched with molybdenum in two consecutive years. In the first year, the seeds of three cultivars, CD-206, MSoy-7101 and BRS-183, were enriched with two foliar applications of 400 g.ha ${ }^{-1}$ of Mo, in stages R3 and R5. Evaluations were made of the yield, thousand seed weight and molybdenum content. The germination and vigor for accelerated ageing were evaluated at zero and after six months storage. In the second year, the cultivars CD-206 and BRS-183, produced from the seeds enriched with Mo the previous year, were treated with Mo at a dosage of $20 \mathrm{~g} \cdot \mathrm{ha}^{-1}$. Evaluations were made of the yield, thousand seed weight, molybdenum concentration and germination and vigor by accelerated ageing. The seed produced by applying molybdenum at high dosages during the R3 and R5 stages showed high levels of the nutrient but did not transfer the same concentration of nutrients to the next seed generation. This enrichment and application via the seeds did not affect the physiological quality of the seed produced or the productivity under the conditions of the study.
\end{abstract}

Index terms: Glycine max, micronutrient, productivity, vigor.

${ }^{1}$ Submetido em 12/11/2009. Aceito para publicação em 15/04/2010. Parte da Tese de Doutorado.

${ }^{2}$ Universidade Tecnológica Federal do Paraná, Campus Dois Vizinhos, Caixa Postal 157, CEP 85660-000, Dois Vizinhos, PR, Brasil. E-mail jpossenti@utfpr.edu.br. (autor para correspondência).

${ }^{3}$ Universidade Federal de Pelotas, Campus Universitário, Caixa Postal 354, CEP 96010-900, Pelotas, RS, Brasil. E-mail francisco.villela@ ufpel.edu.br. 


\section{INTRODUÇÃO}

No processo de produção de sementes, todas as etapas são igualmente importantes para obter se alta qualidade. Campos cultivados com sementes de soja com alto vigor tendem a apresentar melhores índices de produtividade (Kolchinski et al., 2005). Dentre as tecnologias testadas e recomendadas ao produtor de soja está o enriquecimento de sementes com molibdênio, via aplicação foliar, em doses elevadas durante a fase reprodutiva.

O Mo é importante para a atuação da enzima nitrato redutase que é responsável pela redução do nitrato em nitrito no citoplasma celular e por participar do metabolismo do nitrogênio como co-fator das enzimas nitrogenase e redutase do nitrito. É considerado um elemento essencial para as plantas (Martens e Westermann, 1991). Presume-se que, quanto mais eficiente for a assimilação do nitrogênio inorgânico, melhor seja também todas as reações bioquímicas da planta, pela suficiente produção de proteínas e enzimas. É possível que sejam afetadas positivamente as enzimas e proteínas responsáveis pela formação e manutenção das membranas plasmáticas, permitindo assim melhores arranjos de suas estruturas, durante o período de armazenamento e também na germinação. Segundo Trigo et al., (1997), o maior teor de molibdênio nas sementes de soja, possivelmente esteja relacionado com a qualidade fisiológica.

Guerra et al., (2006) avaliando a qualidade fisiológica de sementes de soja em função da adubação com molibdênio, em um Latossolo Vermelho Distroférrico, na região dos Cerrados, concluíram que a aplicação via sementes proporcionou aumento na germinação e emergência de plântulas em campo. Campo e Hungria (2003), enriquecendo sementes de soja com Mo, verificaram aumento no rendimento da cultura. Broch e Ranno (2005) obtiveram ganhos médios de 5,8 sacas de soja por hectare, quando o molibdênio foi aplicado via sementes. Campo e Hungria (2002) concluíram que a melhor resposta foi o enriquecimento de sementes de soja com a dose de 800 gramas de Mo divididas em duas aplicações de 400 gramas cada, nos estágios R3 e R5,4.

$\mathrm{Na}$ bibliografia disponível não se encontrou consenso sobre a ação da aplicação de molibdênio em soja com relação à melhora da qualidade fisiológica das sementes produzidas. $\mathrm{O}$ objetivo do trabalho foi avaliar a qualidade fisiológica e a produtividade de sementes de soja produzidas a partir de sementes enriquecidas com molibdênio, via foliar e via sementes, em dois anos consecutivos.

\section{MATERIAL E MÉTODOS}

O trabalho foi conduzido em área de lavoura de soja na área experimental da Universidade Tecnológica Federal do Paraná (UTFPR), campus de Dois Vizinhos, nos anos agrícolas 2004/2005 e 2005/2006. A região compreende o terceiro planalto paranaense, com altitude de $520 \mathrm{~m}$, latitude de " $25^{\circ} 44^{\prime}$ " Sul e longitude de " 53 '04" Oeste, o clima é classificado como subtropical úmido mesotérmico (Cfa), segundo a classificação de Köppen (Maak, 1968). O solo pertence à Unidade de mapeamento Nitossolo Vermelho distroférrico úmbrico, textura argilosa fase floresta subtropical perenifólia, relevo ondulado (Bhering et al., 2008).

No primeiro ano, o experimento foi implantado no dia 22 de outubro de 2004 e colhido em 19 de março de 2005. No segundo ano, o experimento foi semeado no dia 02 de novembro de 2005 e colhido em 27 de março de 2006. Foram usadas três cultivares de soja CD-206, BRS-183 e MSOY 7101, recomendadas para o estado do Paraná (Embrapa, 2004). As sementes foram tratadas com o inseticida Tiametoxan 350 g. $\mathrm{L}^{-1}$ na dose de 200 $\mathrm{mL} .100 \mathrm{~kg}^{-1}$ de sementes e o fungicida Fluodioxonil 25 g.L.-1 + Metalaxil-M 10 g.L $\mathrm{L}^{-1}$ na dose de $100 \mathrm{~mL} .100 \mathrm{~kg}^{-1} \mathrm{e}$ inoculadas com inoculante em gel com $5 \times 10^{9}$ rizóbios por miligrama, na dose $60 \mathrm{~mL} .50 \mathrm{~kg}^{-1}$ de semente. $\mathrm{O} \mathrm{pH}$ do solo, determinado pelo método de cloreto de cálcio, estava em 5,5 e em água 5,0. Está apresentado na Tabela 1, o resultado da análise de solo realizada antes da semeadura da primeira fase do experimento.

Os elementos $\mathrm{P}, \mathrm{K}^{+}, \mathrm{Cu}, \mathrm{Zn}, \mathrm{Fe}$ e $\mathrm{Mn}$ foram extraídos por Mehllich I, $\mathrm{Ca}^{2+}, \mathrm{Mg}^{2+}, \mathrm{Al}^{3+}$, extraídos por $\mathrm{KCl}$; $\mathrm{C}$, extraído por Walkey Black; $\mathrm{pH}$, pelo método de Cloreto de Cálcio; $\mathrm{S}-\left(\mathrm{SO}_{4}\right)^{2}$, extraído pelo método do Fosfato Monocálcico e B por Cloreto de Bário.

Implantou-se o experimento com semeadora adubadora tratorizada, com nove linhas espaçadas em 0,4 metros e adubação de $350 \mathrm{~kg} \cdot \mathrm{ha}^{-1}$ da fórmula 00 20-20 (N- $\left.\mathrm{P}_{2} \mathrm{O}_{5}-\mathrm{K}_{2} \mathrm{O}\right)$, mistura de grânulos. As unidades experimentais constituíram-se de 24 parcelas com dimensões de quatro metros de largura por 18 metros de comprimento, totalizando 72 metros quadrados por parcela. Colheram-se em cada parcela, 5 linhas com 10 metros de comprimento, totalizando 28 metros quadrados, constituindo-se nas unidades de observação. 
Usou-se o delineamento experimental de blocos casualizados com dois fatores, em um esquema fatorial de $3 \times 2$ com quatro repetições. Os fatores foram três cultivares de soja, CD-206, BRS-183 e MSOY 7101 e o enriquecimento das sementes com molibdênio, com os níveis presença ou ausência. Obteve-se então, 6 tratamentos consistindo os mesmos das combinações entre os níveis dos fatores.

TABELA 1. Resultado da análise de solo, realizada antes da semeadura da primeira fase do experimento, na profundidade de 0 a $20 \mathrm{~cm}$.

\begin{tabular}{|c|c|c|c|c|c|c|c|c|}
\hline Elemento & $\begin{array}{c}\mathrm{P} \\
\left(\mathrm{mg} / \mathrm{dm}^{3}\right)\end{array}$ & $\begin{array}{c}\mathrm{K}^{+} \\
\left(\mathrm{cmol}_{\mathrm{c}} / \mathrm{dm}^{3}\right)\end{array}$ & $\begin{array}{c}\mathrm{C} \\
\left(\mathrm{g} / \mathrm{dm}^{3}\right)\end{array}$ & $\begin{array}{c}\mathrm{Ca}^{2+} \\
\left(\mathrm{cmol}_{\mathrm{c}} / \mathrm{dm}^{3}\right)\end{array}$ & $\begin{array}{c}\mathrm{Mg}^{2+} \\
\left(\mathrm{cmol}_{\mathrm{c}} / \mathrm{dm}^{3}\right)\end{array}$ & $\begin{array}{l}\mathrm{S}-\left(\mathrm{SO}_{4}\right)^{2-} \\
\left(\mathrm{mg} / \mathrm{dm}^{3}\right)\end{array}$ & $\mathrm{pH}$ & $\begin{array}{c}\mathrm{Al}^{3+} \\
\left(\mathrm{cmol}_{\mathrm{c}} / \mathrm{dm}^{3}\right)\end{array}$ \\
\hline Valor & 6,30 & 0,15 & 16,69 & 3,97 & 2,44 & 8,77 & 4,90 & 0,00 \\
\hline Elemento & $\begin{array}{c}\mathrm{Cu} \\
\left(\mathrm{mg} / \mathrm{dm}^{3}\right)\end{array}$ & $\begin{array}{c}\mathrm{Zn} \\
\left(\mathrm{mg} / \mathrm{dm}^{3}\right)\end{array}$ & $\begin{array}{c}\mathrm{Fe} \\
\left(\mathrm{mg} / \mathrm{dm}^{3}\right)\end{array}$ & $\begin{array}{c}\mathrm{Mn} \\
\left(\mathrm{mg} / \mathrm{dm}^{3}\right)\end{array}$ & $\begin{array}{c}\text { B } \\
\left(\mathrm{mg} / \mathrm{dm}^{3}\right)\end{array}$ & & & \\
\hline Valor & 6,52 & 1,96 & 60,00 & 86,00 & 0,18 & & & \\
\hline
\end{tabular}

O enriquecimento das sementes, realizado no primeiro ano de implantação do experimento, foi com 800 g.ha- ${ }^{-1}$ de molibdênio, dividido em duas aplicações de molibdato de sódio a 39\% usando pulverizador costal propelido com $\mathrm{CO}_{2}$. A primeira aplicação foi na fase reprodutiva $\mathrm{R} 3$ (Fehr et al., 1971), em 22 de janeiro de 2005, na dose de 400 g.ha $^{-1}$ e a segunda em 02 de fevereiro, também na dose de 400 g.ha $^{-1}$, na fase de R5 (Fehr et al., 1971). As variáveis analisadas foram produtividade, peso de mil sementes (PMS), teor de molibdênio nas sementes, germinação e vigor por envelhecimento acelerado. Avaliou-se a germinação de acordo com as Regras para Análise de Sementes, (Brasil, 2009), usando 4 repetições de 100 sementes em rolo de papel a $25{ }^{\circ} \mathrm{C}$ e o vigor por envelhecimento acelerado conforme descrito por Marcos Filho (1994), submetendo-se as sementes à uma temperatura de $41{ }^{\circ} \mathrm{C}$ por um período de 48 horas. A determinação do Mo, foi baseada no método de análise de tecido vegetal conforme Malavolta et al., (1989). A colheita foi realizada manualmente, na fase R7 (Fehr et al., 1971). A germinação e o envelhecimento acelerado, foram analisados a zero (logo após a colheita) e seis meses de armazenamento das sementes em armazém convencional sob condições naturais.

No segundo ano de condução do experimento, as sementes das cultivares CD-206 e BRS-183, foram semeadas em campo sobre resteva de trigo. A adubação de base foi de $250 \mathrm{~kg} \cdot$ ha $^{-1}$ da fórmula 00-20-20 (N- $\mathrm{P}_{2} \mathrm{O}_{5}$ $\mathrm{K}_{2} \mathrm{O}$ ), mistura de grânulos. As unidades experimentais constaram de 32 parcelas com dimensões de quatro metros de largura, por 10 metros de comprimento, totalizando 40 metros quadrados cada. Usou-se o delineamento experimental de blocos casualizados no esquema fatorial de $2 \times 2 \times 2$, com quatro repetições. Os fatores testados foram: presença ou não do enriquecimento das sementes feito na planta mãe, na geração anterior; o fator cultivar, com as cultivares CD-206 e BRS-183 e, presença ou ausência de Mo, aplicados via semente no momento da semeadura, na dose de $20 \mathrm{~g} \cdot \mathrm{ha}^{-1}$, na forma de molibdênio de sódio a $39 \%$ de Mo. Os tratamentos foram as combinações dos níveis dos fatores.

A colheita também foi realizada manualmente, na fase R7 (Fehr et al., 1971). Avaliou-se a produtividade $\left(\mathrm{kg} \cdot \mathrm{ha}^{-1}\right)$, peso de mil sementes, teor de Mo e a qualidade fisiológica das sementes pelos testes de germinação e vigor por envelhecimento acelerado.

Para os dois anos de condução do experimento, as sementes colhidas foram beneficiadas somente em classificador de espiral, devido ao pequeno volume colhido nas parcelas.

A precipitação pluviométrica no local do experimento foi acompanhada pela instalação de um pluviômetro. Analisaram-se os fatores qualitativamente, com o auxilio do programa para análises estatísticas Sanest (Zonta e Machado, 1984).

\section{RESULTADOS E DISCUSSÃO}

Observou-se para todas as variáveis analisadas precisão experimental, pois os coeficientes de variação 
ficaram abaixo de $20 \%$. Os resultados obtidos para produtividade das três cultivares não apresentaram diferença significativa (Tabela 2). Resultados diferentes foram encontrados por Broch (2004) e Ceretta et al., (2005), que obtiveram incrementos em produtividade em Latossolos Vermelhos Distroférricos. No entanto, os resultados foram coincidentes com Zancanaro et al., $(2003,2004)$ que trabalharam em solo arenoso. O PMS observado para as três cultivares apresentou diferença entre a cultivar BRS-183 com 150,87 gramas contra 135,25 gramas e 135 gramas para CD-206 e MSOY 7101 respectivamente, quando não se enriqueceu as sementes. As sementes enriquecidas não apresentaram diferença significativa entre si no PMS, mas notam-se para as sementes não enriquecidas que as cultivares CD206 e MSOY-7101 apresentaram menor PMS do que a cultivar BRS-183 evidenciando efeito da cultivar e não do enriquecimento em si. Estes resultados concordam com aqueles encontrados por Zancanaro et al. (2003) em solo arenoso. Por outro lado, Barbosa et al. (2005) encontraram resultados positivos entre a aplicação de Mo e peso de mil sementes em feijoeiro. Quanto ao teor de Mo, notouse diferença para as três cultivares quando se enriqueceu as sementes, mostrando que a aplicação deste elemento sobre a planta, por meio de pulverização foliar, na fase reprodutiva, eleva seus teores na semente produzida. As cultivares testadas não apresentaram diferenças entre suas médias de produtividade, quando houve o enriquecimento com Mo, podendo ser questionado o custo benefício desta prática do ponto de vista econômico. Antes da semeadura, as três cultivares testadas, apresentaram níveis de molibdênio abaixo do limite de detecção do teste.

TABELA 2. Produtividade, peso de mil sementes e teor de molibdênio das sementes de três cultivares de soja, enriquecidas ou não com Mo.

\begin{tabular}{|c|c|c|c|}
\hline \multirow{2}{*}{ Cultivar } & \multicolumn{3}{|c|}{ Produtividade $\left(\mathrm{kg} \cdot \mathrm{ha}^{-1}\right)$} \\
\hline & S/Enriq & C/Enriq & Média \\
\hline CD-206 & $2.859,1$ & $2.825,7$ & $2.842,4 \mathrm{~A}^{1}$ \\
\hline MSOY-7101 & $2.820,6$ & $2.804,6$ & $2.812,6 \mathrm{~A}$ \\
\hline BRS-183 & $2.784,8$ & $2.616,0$ & $2.700,4 \mathrm{~A}$ \\
\hline Média & $2.821,5 \mathrm{a}$ & $2.748,8 \mathrm{a}$ & \\
\hline $\mathrm{CV}(\%)$ & 7,8 & & \\
\hline \multicolumn{4}{|c|}{ Peso de mil sementes $(\mathrm{g})$} \\
\hline CD-206 & $135,25 \mathrm{~B}$ & $137,87 \mathrm{~A}$ & 136,56 \\
\hline MSOY-7101 & $135,00 \mathrm{~B}$ & $138,50 \mathrm{~A}$ & 136,75 \\
\hline BRS-183 & $150,87 \mathrm{~A}$ & $139,87 \mathrm{~A}$ & 145,37 \\
\hline Média & $140,37 \mathrm{a}$ & $138,75 \mathrm{a}$ & \\
\hline $\mathrm{CV}(\%)$ & 3,5 & & \\
\hline \multicolumn{4}{|c|}{ Teor de molibdênio (ppm) } \\
\hline CD-206 & *nd & 31,53 & $15,76 \mathrm{~A}$ \\
\hline MSOY-7101 & 0,14 & 34,68 & $17,34 \mathrm{~A}$ \\
\hline BRS-183 & 0,63 & 35,30 & $17,65 \mathrm{~A}$ \\
\hline Média & $0,21 \mathrm{~b}$ & $33,84 \mathrm{a}$ & \\
\hline $\mathrm{CV}(\%)$ & 9,4 & & \\
\hline
\end{tabular}

${ }^{1}$ Médias seguidas por mesma letra, maiúscula na coluna e minúscula na linha, não diferem entre si, pelo teste de Duncan a $1 \%$ de probabilidade d o erro.

Para a variável germinação (Tabela 3), não se evidenciou efeito positivo do enriquecimento das sementes com Mo, ao serem comparadas as enriquecidas com aquelas obtidas das parcelas que não foram enriquecidas. Após 
seis meses de armazenamento e próximo à semeadura do segundo ano, as médias de todas as cultivares apresentaramse abaixo do mínimo aceitável comercialmente. Guerra et al., (2006), verificaram aumento na porcentagem de germinação de sementes de soja, mediante a aplicação do elemento via sementes por ocasião da semeadura.

TABELA 3. Germinação e vigor, avaliado pelo teste de envelhecimento acelerado, logo após a colheita e aos seis meses de armazenamento de sementes de três cultivares de soja, enriquecidas ou não com Mo.

\begin{tabular}{|c|c|c|c|c|c|c|}
\hline \multirow{2}{*}{ Cultivar } & \multicolumn{3}{|c|}{ Após a colheita } & \multicolumn{3}{|c|}{ Aos seis meses } \\
\hline & Ñ. Enriq & Enriq & Média & N. Enriq & Enriq & Média \\
\hline \multicolumn{7}{|c|}{ Germinação (\%) } \\
\hline CD-206 & 93 & 91 & $92 \mathrm{~A}^{1}$ & 68 & 69 & $69 \mathrm{~A}$ \\
\hline MSOY-7101 & 81 & 81 & $81 \mathrm{~B}$ & 47 & 52 & $50 \mathrm{~B}$ \\
\hline BRS-183 & 94 & 93 & $94 \mathrm{~A}$ & 77 & 73 & $75 \mathrm{~A}$ \\
\hline Média & 89 a & $88 \mathrm{a}$ & & $64 \mathrm{a}$ & $65 \mathrm{a}$ & \\
\hline $\mathrm{CV}(\%)$ & & 3,5 & & & 9,2 & \\
\hline \multicolumn{7}{|c|}{ Envelhecimento acelerado (\%) } \\
\hline CD-206 & $79 \mathrm{~A}$ & $79 \mathrm{AB}$ & 79 & 41 & 43 & $42 \mathrm{~A}$ \\
\hline MSOY-7101 & $65 \mathrm{~B}$ & $71 \mathrm{~B}$ & 68 & 18 & 21 & $19 \mathrm{~B}$ \\
\hline BRS-183 & $84 \mathrm{~A}$ & $86 \mathrm{~A}$ & 85 & 48 & 40 & $44 \mathrm{~A}$ \\
\hline Média & $76 \mathrm{a}$ & $79 \mathrm{a}$ & & $36 \mathrm{a}$ & $35 \mathrm{a}$ & \\
\hline $\mathrm{CV}(\%)$ & & 5,7 & & & 20,0 & \\
\hline
\end{tabular}

${ }^{1}$ Médias seguidas por mesma letra, maiúscula na coluna e minúscula na linha, não diferem entre si, pelo teste de Duncan a $1 \%$ de probabilidade do erro.

O teste de envelhecimento acelerado, realizado logo após a colheita, apresentou diferenças significativas entre as cultivares. Contudo, não se verificou o efeito do enriquecimento das sementes com Mo mediante a pulverização das parcelas com este elemento nos resultados deste teste de vigor. Após seis meses de armazenamento, o teste de envelhecimento acelerado, revelou que todas as cultivares testadas mantiveram a mesma tendência, mas diminuindo a sua performance. Não se notou melhora no desempenho deste teste em função do enriquecimento das sementes.

Há de ser considerado, que durante a maturação fisiológica das sementes, no período compreendido entre os meses de janeiro e fevereiro de 2005 , a precipitação pluvial foi 59 e 71 milímetros, respectivamente. Este fato pode ajudar a explicar os baixos valores obtidos para germinação e vigor por envelhecimento acelerado, para todos os tratamentos, Além do que o volume de sementes colhido nas parcelas, impossibilitou o beneficiamento em máquina de ar e peneiras e mesa de gravidade.

$\mathrm{Na}$ segunda etapa do experimento, verificou-se que não ocorreu diferença significativa entre as médias das cultivares, ao ser adicionado Mo via semente por ocasião da semeadura e tampouco, quando esse nutriente não foi adicionado à semente com relação à produtividade e peso de mil sementes (Tabela 4). $\mathrm{O}$ processo de enriquecimento das sementes com Mo na geração anterior, não contribuiu para aumentos em produtividade, adicionando-se ou não este elemento às sementes por ocasião da semeadura no ano seguinte. Os resultados discordam daqueles obtidos por Broch (2004), que encontrou resposta significativa na produtividade de soja enriquecida com molibdênio na geração anterior. O PMS apresentou efeito significativo entre as cultivares, porém também não houve resposta significativa do enriquecimento das sementes com Mo na geração anterior e nem da adição de Mo via semente por ocasião da semeadura. 
TABELA 4. Produtividade, peso de mil sementes, germinação e emergência de plântulas em campo de duas cultivares de soja, oriundas de sementes enriquecidas ou não com Mo, com e sem aplicação de Mo via semente.

\begin{tabular}{|c|c|c|c|c|c|c|}
\hline \multicolumn{7}{|c|}{ Produtividade $\left(\mathrm{kg} \cdot \mathrm{ha}^{-1}\right)$} \\
\hline \multirow{2}{*}{ Cultivar } & \multicolumn{2}{|c|}{ S/Mo via semente } & \multirow{2}{*}{ Média } & \multicolumn{2}{|c|}{ C/Mo via semente } & \multirow{2}{*}{ Média } \\
\hline & Ñ. Enriq. & Enriq. & & N. Enriq. & Enriq. & \\
\hline CD-206 & $2.973,7$ & $3.203,4$ & $3.088,5 \mathrm{~A}^{1}$ & $3.212,6$ & $3.313,6$ & $3.263,1 \mathrm{~A}$ \\
\hline BRS-183 & $3.105,4$ & $3.102,3$ & $3.103,9 \mathrm{~A}$ & $3.035,0$ & $2.894,1$ & $2.964,5 \mathrm{~A}$ \\
\hline Média & $3.039,5 \mathrm{a}$ & $3.152,8 \mathrm{a}$ & & $3.103,9 \mathrm{a}$ & $3.123,7 \mathrm{a}$ & \\
\hline $\mathrm{CV} \%$ & \multicolumn{6}{|c|}{12,2} \\
\hline \multicolumn{7}{|c|}{ Peso de mil sementes (g) } \\
\hline CD-206 & $161,62 \mathrm{~A}$ & $162,57 \mathrm{~A}$ & 162,10 & $163,15 \mathrm{~A}$ & $161,67 \mathrm{~A}$ & 162,41 \\
\hline BRS-183 & $155,50 \mathrm{~A}$ & $155,17 \mathrm{~B}$ & 155,36 & $156,52 \mathrm{~B}$ & $154,90 \mathrm{~B}$ & 155,70 \\
\hline Média & $158,58 \mathrm{a}$ & $158,87 \mathrm{a}$ & & $159,84 \mathrm{a}$ & $158,28 \mathrm{a}$ & \\
\hline $\mathrm{CV} \%$ & \multicolumn{6}{|c|}{1,9} \\
\hline
\end{tabular}

Germinação (\%)

\begin{tabular}{|c|c|c|c|c|c|c|}
\hline \multirow{2}{*}{ Cultivar } & \multicolumn{2}{|c|}{ S/Mo via semente } & \multirow{2}{*}{ Média } & \multicolumn{2}{|c|}{ C/Mo via semente } & \multirow{2}{*}{ Média } \\
\hline & Ñ. Enriq. & Enriq. & & Ñ. Enriq. & Enriq. & \\
\hline CD-206 & 78 & 78 & $78 \mathrm{~A}$ & 73 & 73 & $73 \mathrm{~B}$ \\
\hline BRS-183 & 86 & 80 & $87 \mathrm{~A}$ & 86 & 88 & $87 \mathrm{~A}$ \\
\hline Média & $82 \mathrm{a}$ & $79 \mathrm{a}$ & & $80 \mathrm{a}$ & $80 \mathrm{a}$ & \\
\hline $\mathrm{CV} \%$ & \multicolumn{6}{|c|}{9,7} \\
\hline \multicolumn{7}{|c|}{ Envelhecimento acelerado (\%) } \\
\hline CD-206 & $59 \mathrm{~B}$ & $69 \mathrm{~A}$ & 64 & 65 & 68 & $66 \mathrm{~A}$ \\
\hline BRS-183 & $74 \mathrm{~A}$ & $70 \mathrm{~A}$ & 72 & 73 & 77 & $75 \mathrm{~A}$ \\
\hline Média & $67 \mathrm{a}$ & $69 a$ & & $69 a$ & $72 \mathrm{a}$ & \\
\hline $\mathrm{CV} \%$ & & & & 9 & & \\
\hline
\end{tabular}

${ }^{1}$ Médias seguidas por mesma letra, maiúscula na coluna e minúscula na linha, não diferem entre si, pelo teste de Duncan a $1 \%$ de probabilidade do erro.

O teste de germinação revelou que o molibdênio aplicado na semente no momento da semeadura, possibilitou valores de germinação de 86 e $88 \%$ para sementes produzidas a partir de sementes não enriquecidas e enriquecidas na geração anterior, respectivamente para a cultivar BRS-183 (Tabela 4). Especificamente, a diferença entre as cultivares apareceu nas médias das parcelas em que foram semeadas com sementes enriquecidas com Mo, de $87 \%$ para BRS-183 e $73 \%$ para CD-206. Os resultados do teste de envelhecimento acelerado não apresentam diferenças entre os tratamentos aplicados, evidenciando que o enriquecimento das sementes, não representou efeito significativo para este teste. Quando se analisou o teor de molibdênio para as sementes colhidas no segundo ano do experimento, os valores ficaram abaixo do nível mínimo de detecção do teste, para todos os tratamentos oriundos de sementes enriquecidas ou não na geração anterior e também para quando se adicionou o micronutriente via semente. Estes dados coincidiram com aqueles encontrados por Gurley e Giddens (1969) que verificaram pequena transferência de Mo da semente original para a segunda geração e que na terceira geração, o teor deste nutriente deixou de existir.

Portanto, apesar de efeitos relativos às cultivares, o 
enriquecimento das sementes com molibdênio não resultou em aumentos no rendimento ou mesmo na qualidade fisiológica das sementes produzidas, para os dois anos de condução do experimento.

\section{CONCLUSÕES}

Apesar de terem sido resultados obtidos em determinadas condições de clima e solo, que não são definitivos e não esgotam o assunto a que se propôs pesquisar, pode-se concluir que:

- A semente produzida mediante a aplicação de Mo em altas doses, durante as fases de R3 e R5, apresenta altas concentrações do micronutriente, mas o enriquecimento não transferiu Mo para a semente da próxima geração, em níveis detectáveis;

- A aplicação de Mo via semente e/ou via foliar não afeta a qualidade fisiológica das sementes produzidas assim como a produtividade e o peso de mil sementes.

\section{REFERÊNCIAS}

BARBOSA, G.F.; ARF, O.; DE SÁ, M.E.; DO NASCIMENTO, M.S., ORIOLI JÚNIOR, V.; FONSECA, A.E.; COSTA, R.S.S. Efeitos de doses de nitrogênio e de molibdênio na qualidade fisiológica das sementes e produtividade do feijoeiro (Phaseolus vulgaris) de inverno em sistema de plantio direto. Informativo ABRATES, v.15, n.1/2/3, p.106, 2005.

BHERING, S.B.; SANTOS, H.G. dos. Mapa de solos do Estado do Paraná: legenda atualizada. Rio de Janeiro: Embrapa Solos: Rio de Janeiro Florestas, Colombo, PR, 2008, 74p.

BRASIL. Ministério da Agricultura, Pecuária e Abastecimento. Regras para análise de sementes. Ministério da Agricultura, Pecuária e Abastecimento. Secretaria de Defesa Agropecuária. Brasília, DF: MAPA/ ACS, 2009. 395p.

BROCH, D.L. Efeito do enriquecimento de sementes com molibdênio na planta mãe, sobre a produtividade de soja. Maracajú, MS: Fundação MS, 2004. 5p. (Informativo Técnico 01/2004).

BROCH, D.L.; RANNO, S.K. Efeito do teor de molibdênio nas sementes e da aplicação de molibdênio e cobalto via sementes sobre a produtividade da soja na safra 2004/05. Maracajú, MS: Fundação MS, 2005b. 10p. (Informativo Técnico 02/2005).

CAMPO, R. J.; HUNGRIA, M. Enriquecimento de sementes de soja com molibdênio como fator de aumento da eficiência da fixação biológica do nitrogênio e do rendimento da soja. In: REUNIÃO DE PESQUISA DE SOJA DA REGIÃ̃O CENTRAL DO BRASIL, 25., 2003, Uberaba. Resumos... Londrina: Embrapa Soja, EPAMIG: Fundação Triângulo, 2003. p.156-157.

CAMPO, R.J.; HUNGRIA, M. Importância dos micronutrientes na fixação biológica do nitrogênio. In: CONGRESSO BRASILEIRO DE SOJA, 2., MERCOSOJA 2002, Foz do Iguaçu. Anais... (SARAIVA, O.F.; HOFFMAN-CAMPO, B. (Org.). Perspectivas do Agronegócio da Soja. Londrina: Embrapa Soja, 2002. p.355-366.

CERETTA, C.A.; PAVINATTO, A.; PAVINATTO, P.S.; MOREIRA, I.C.L.; GIROTTO, E, TRENTIN, E. E. Micronutrientes na soja: produtividade e análise econômica. Ciência Rural, v.35, n.3, p. 576-581, 2005.

EMBRAPA. Tecnologias de produção de soja - Paraná 2005. Londrina: Embrapa Soja, 2004. 224p. (Embrapa Soja. Sistema de Produção, 5).

FEHR, W.R; CAVINESS, C.E.; BURMOOD, D.T. PENNINGTON, J.S. Stage of development description form soybeans, Glycine max (L.) Merrill. Crop Science, v.11, p.929-931, 1971.

GUERRA, C.A.; MARCHETTI, M.E.; ROBAINA, A.D.; DE SOUZA, C.F.; GONÇALVES, M.C.; NOVELINO, J.O. Soybean seed physiological quality in function of phosphorus, molybdenum and cobalt fertilization. Acta Scientarum Agronomy, v.28, n.1, 2006. Disponível na Internet: <http://www.eduem.uem.br/acta/ agro/2006_1/14_150_05.pdf>. Acesso em: 15 jan. 2007.

GURLEY, W.H.; GIDDENS, J. Factors affecting uptake, yield response, and carryover of molybdenum in soybean seed. Agronomy Journal, v.61, n.1, p.7-9, 1969.

KOLCHINSKI, E.M.; SCHUCH, L.O.B.; PESKE, S.T. Seeds vigor and intra-specific competition in soybean. Ciência Rural, v.35, n.6, p.1248-1256, 2005.

MAAK, R. geografia física do estado do Paraná. Curitiba: Banco de desenvolvimento do Paraná, 1968. 350 p.

MALAVOLTA, E.; VITTI, G.C.; OLIVEIRA, S.A. Avaliação do estado nutricional das plantas; princípios e aplicações. Piracicaba: Assoc. Bras. Pesq. Potássio e do Fosfato, 1989. 201p. 
MARCOS FILHO, J. Teste de envelhecimento acelerado. In: VIEIRA, R.P.; CARVALHO, N.M. Testes de vigor em sementes. Jaboticabal: Funep, 1994. p.133-149.

MARTENS, D.C; WESTERMANN, D.T. Fertilizers applications for correcting micronutrient deficiencies. In: MORTVEDT, JJ.; COX, F.R.; SHUMAN, L.M.; WELCH, R.M. (Ed.). Fertilizers applications for correcting micronutrient deficiencies: micronutrients in agriculture. 2.ed. Madison: Soil Science Society of America, 1991. p.549-592.

TRIGO, L.F.N.; PESKE, S.T.; GASTAL, M.F. C.; VAHL, L.C.; TRIGO, M.F. O. Efeito do conteúdo de fósforo na semente de soja sobre o rendimento da planta resultante. Revista Brasileira de Sementes, v.19, n.1, p.111-115,
1997.

ZANCANARO, L.; TESSARO, L.C.; HILLESHEIM, J. Relatório Final Projeto Específico Soja. Rondonópolis, MT : Fundação MT, 2003. 7p. (Informativo Técnico 01/2003).

ZANCANARO, L.; TESSARO, L.C.; HILLESHEIM, J. Relatório Final Projeto Específico Soja. Rondonópolis, MT : Fundação MT, 2004. 8p. (Informativo Técnico 01/2004).

ZONTA, E.P.; MACHADO, A.A. Sistema de análise estatística para microcomputadores-SANEST. Pelotas: UFPel, Instituto de Física e Matemática, 1984. 150p. 